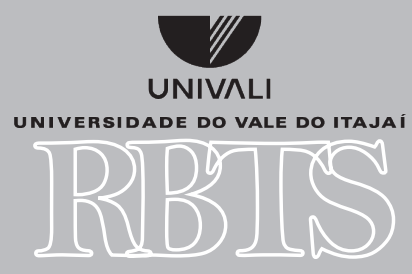

Revista Brasileira de Tecnologias Sociais
1 Graduação em Nutrição pela Universidade do Vale do Itajaí. Especialista em Nutrição Clínica Funcional pelo Centro Valéria Paschoal de Educação, Mestre em Ciências Farmacêuticas pela Universidade do Vale do Itajaí. E-mail: claiza@univali.br

2 Graduação em Psicologia pela Faculdade Paulistana de Ciências e Letras. Mestrado em Psicologia Pontifícia Universidade Católica do Rio Grande do Sul. moliveira@univali.br

3 Graduação em Fisioterapia pela Universidade Tuiuti do Paraná. Especialização em Cardiorrespiratória pela Universidade Tuiuti. Mestrado em Saúde e Gestão do Trabalho pela Universidade Univali. E-mail: alexsandradias@univali.br

4. Graduação em Fisioterapia pela Universidade Federal de Santa Catarina. Especialização em Cardiorrespiratória pela Universidade Tuiuti. Mestrado em Saúde e Gestão do Trabalho pela Universidade Univali. Doutorado em Educação Científica e Tecnológica pela Universidade Universidade Federal de Santa Catarina.E-mail: fabiola.chesani@ univali.br

\section{Caracterização dos cuidadores das crianças e dos adolescentes atendidos pelo projeto de extensão de um hospital universitário infantil}

\section{Caracterization of caregivers of children and adolescents served by the extension project of a university children's hospital}

\author{
Clatiza Barreta ${ }^{1}$; Márcia Aparecida Miranda de Oliveira²; Alessandra Marinho \\ Dias $^{3} \&$ Fabíola Hermes Chesant ${ }^{4}$
}

RESUMO: Este estudo objetivou caracterizar os cuidadores das crianças e dos adolescentes participantes do projeto de extensão em um hospital universitário infantil, localizado no litoral catarinense. Realizou-se um estudo quantitativo de caráter descritivo em um hospital universitário infantil, localizado em um município catarinense. Foi realizada a aplicação de um formulário embasado no instrumento de avaliação de condição de saúde. As variáveis coletadas a partir destes formulários constituíram questões de identificação, características socioeconômicas, hábitos de vida e saúde. A amostra da pesquisa foi de 100 cuidadores de ambos os sexos. Os mesmos foram tabulados e analisados no programa Microsoft Excel. Por meio dos resultados foi possível perceber que $91 \%$ dos cuidadores eram do sexo feminino; a maioria dos entrevistados não pratica nenhum tipo de exercício físico (63\%); possui histórico de doença familiar (61\%), principalmente diabetes (36\%); não fazem uso de bebidas alcoólicas ( $80 \%$ ) e não são tabagistas ( $86 \%$ ) e uma parcela significativa (28\%) faz uso de medicamentos de uso contínuo, entre os quais os antidepressivos aparecem com maior frequência.

PALAVRAS-CHAVE: Perfil de saúde. Cuidadores. Hospitalização. Educação em saúde. Humanização da assistência.

\section{ABSTRACT}

This study aimed to characterize caregivers of children and adolescents participating in the extension project at a children's university hospital, located on the Santa Catarina coast. We conducted a quantitative, descriptive study in a university children's hospital located in a municipality of Santa Catarina. A form was applied, based on the tool to assess health condition. The variables collected through these forms consisted of identification questions, socioeconomic characteristics, lifestyle habits, and health. The survey sample included one hundred caregivers of both sexes. The data were tabulated and analyzed in a Microsoft Excel spreadsheet. The results showed that $91 \%$ of the caregivers were women, the majority of the respondents did not practice any physical exercise $(63 \%)$, had a family history of disease (61\%), especially diabetes $(36 \%)$, did not 
use alcohol (80\%) and were nonsmokers (86\%), and a significant portion (28\%) were in continuous use of medications, the main one being antidepressants.

KEYWORDS: Health profile; caregivers; hospitalization; health education; humanization of assistance.

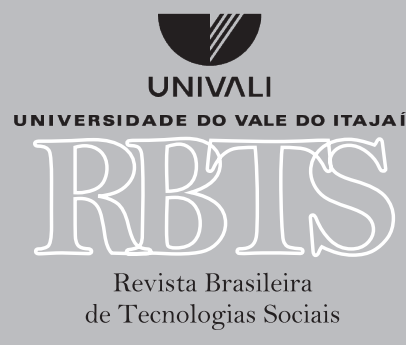

Revista Brasileira

de Tecnologias Sociais 
O Plano Nacional de Extensão Universitária (2003) teve como meta fomentar projetos que visam proporcionar mudanças no cenário social ao articular o tripé ensino, pesquisa e extensão. Os projetos de extensão devem perpassar os muros da universidade, indo ao encontro da comunidade, favorecendo a identificação de situações de risco e de estratégias para o seu enfrentamento. Nesta perspectiva, a universidade, representada por acadêmicos e docentes, e a comunidade devem se envolver. Foi com este pensamento que, em 2004, a Universidade do Vale do Itajaí (UNIVALI) criou um programa de extensão universitária denominado "Para Saber Viver", sendo que um dos seus eixos apontava para os cuidados com a saúde da criança e do adolescente. Visando atender a uma necessidade emergente do hospital pediátrico, surgiu o projeto "Humanizar e Educar em Saúde”, desenvolvido no Hospital Universitário Pequeno Anjo (HUPA), junto aos usuários do Sistema Único de Saúde (SUS), que até o presente momento contemplou cerca de 3.500 pessoas.

O projeto é constituído por uma equipe interdisciplinar, cujos docentes e discentes dos cursos de Fisioterapia, Psicologia, Odontologia e Nutrição reúnem-se diariamente com cuidadores de crianças e adolescentes hospitalizados ou em atendimento ambulatorial para socializar conhecimentos e favorecer a troca de saberes de acordo com os pressupostos da humanização e de Paulo Freire.

O projeto de extensão tem suas ações voltadas para os cuidadores e familiares das crianças ou adolescentes, alvos do programa de humanização, pois são eles que acompanham o sofrimento causado pela doença, assim como colaboram para a melhoria da qualidade de vida. A educação em saúde tem o papel de promover uma decisão informada à população sobre os riscos à saúde a que estão expostos, considerando seus hábitos de vida e delegando a estes a responsabilidade, na intenção de mudança dos comportamentos de riscos. Porém, a forma simplista centrada em práticas educativas, tratadas na exterioridade dos problemas de saúde, poderá comprometer os resultados. As opções, os desejos, as necessidades, os estilos de vida, as crenças e os valores, muitas vezes causas dos problemas de saúde, requerem ações socialmente sustentadas do ponto de vista político, econômico e cultural.

Neste sentido, a educação em saúde passa a ser o elo integrador entre o saber técnico e o saber popular marcado por uma colaboração mútua que atinge e reorienta a diversidade de práticas realizadas nos serviços de saúde. Mais do que um componente da atenção primária à saúde, exerce função de encorajamento e apoio para que pessoas e grupos sociais assumam maior controle sobre sua saúde e suas vidas (VASCONCELOS, 2001).

Salienta-se também que é perspectiva do projeto promover o trabalho interdisciplinar entre professores dos diversos cursos da saúde e respectivos acadêmicos, assim como contribuir para a consolidação dos projetos pedagógicos dos cursos envolvidos, a partir do desenvolvimento de competências e habilidades gerais constantes nas diretrizes curriculares para os profissionais da saúde, tais como comunicação, liderança e tomada de decisão. Em outras palavras, há uma colaboração para um maior aprendizado de todos, pois há trocas de saberes entre os acadêmicos e os professores dos cursos envolvidos (Fisioterapia, Psicologia, Odontologia e Nutrição).

A proposta do projeto "Humanizar e Educar em Saúde” aspira pelo nascimento de uma nova imagem profissional, responsável pela efetiva promoção da saúde, ao considerar o paciente em sua integridade física, psíquica e social, e não somente de um ponto de vista biológico (CASSEL; HAHN; WULFF apud CAPRARA; FRANCO, 1999). 
Neste sentido o projeto de extensão achou pertinente caracterizar os cuidadores das crianças e dos adolescentes atendidos em um hospital universitário infantil, localizado em um município catarinense, visto que, todos os anos, mais de um milhão de crianças no Brasil são hospitalizadas por diferentes causas. A hospitalização de uma criança criticamente enferma causa na família momentos de angústia, sofrimento e desespero. No contexto, a família vivencia uma ruptura em sua estrutura e funcionamento, na qual os pais percebem perder o poder sobre a criança, que passa a pertencer temporariamente à equipe da unidade (NETO, 2000).

Diante do adoecimento do filho e dos muitos momentos difíceis que enfrentam, os pais acabam necessitando de assistência médica, psicológica, nutricional, fisioterápica, entre outras. Embora os cuidadores reconheçam a importância da participação deles durante o tratamento, eles podem manifestar sobrecarga em decorrência do cuidado prestado constantemente à criança/ adolescente (ORTIZ; LIMA, 2007; MOREIRA; ANGELO, 2008; CAMARGOS et al., 2009).

Os atributos definidores desta vulnerabilidade estão relacionados ao contexto da doença que gera incerteza, impotência, ameaça real ou imaginária, exposição ao dano, temor do resultado, submissão ao desconhecido e expectativas de retornar à vida anterior (CÔA; PETTENGILL, 2011).

Permitir que a família se mantenha fortalecida e autônoma na situação de crise é um dos princípios fundamentais do cuidar, que envolve dimensões de respeito ao ser humano, cujo princípio máximo deve emanar os preceitos éticos de tudo e todos que com esse ser lidam (HAINES; CHILDS, 2005).

A relevância da pesquisa se baseia no fato de que nas bases de dados brasileiras consultadas não foi encontrado um grande número de pesquisas envolvendo a caracterização de crianças e adolescentes, considerando que a caracterização dessa população se faz importante para elaborar e implementar estratégias de intervenção para com os mesmos. Mediante o exposto, o presente trabalho objetiva caracterizar os cuidadores das crianças e dos adolescentes atendidos em um hospital universitário infantil, localizado em um município catarinense.

\section{MATERIAis E MÉTODOS}

Realizou-se um estudo quantitativo de caráter descritivo em um hospital universitário infantil, localizado em um município catarinense. A população envolvida foi de cuidadores que acompanhavam crianças e adolescentes internados nas alas A e B do hospital. A amostra da pesquisa foi de 100 cuidadores de ambos os sexos, tendo como fatores de exclusão: aceitar participar da pesquisa e possuírem idade superior a 18 anos.

Foi aplicado um formulário pelos pesquisadores, no mês de novembro de 2012 e as variáveis coletadas a partir destes formulários constituíram questões de identificação (nome, sexo, idade, cidade em que reside, bairro em que reside, há quanto tempo reside na cidade, cor e estado civil), características socioeconômicas (renda, escolaridade, profissão, número de pessoas na família, parentesco com o hospitalizado, quantidade de cuidadores do acompanhante e há quantos dias estava acompanhando o hospitalizado) e hábitos de vida e saúde (tabagismo, uso de bebida alcoólica, prática de exercício físico, uso de medicamentos contínuos, histórico de doença na família e como considerava a própria saúde).

Os dados coletados foram tabulados com o auxílio do programa Microsoft Excel e foram calculados os percentuais, as médias e os desvios padrão para posterior análise dos resultados. 
Dos 100 cuidadores que acompanham as crianças, noventa e um (91\%) eram do sexo feminino e nove $(9 \%)$ do sexo masculino. Com base nos dados, pode-se observar que a mulher é a principal cuidadora, fator este que está ligado diretamente ao papel social delegado a ela. Para compreender essa construção, devem-se considerar as variáveis históricas que permitem tomar a mulher como a responsável pelos cuidados infantis.

A trajetória histórica da figura da mulher é decorrente de parâmetros culturais já enraizados e socialmente construídos, cujo papel de cuidar é uma atribuição feminina natural. À mulher foi destinado o espaço privado e ao homem o espaço público, quando um familiar necessita de cuidado, é como se ocorresse um despertar dos fatores causais da atribuição feminina, aqueles tradicionais, como tomar conta, cuidar e tratar (BECK; LOPES, 2007). Sendo assim, é mais comum a mulher faltar ao trabalho do que o homem nos casos em que um membro da família necessita de um cuidador (MACHADO et al., 2006).

\section{PERFIL SOCIOECONÔMICO}

Ao perguntar quanto à cor, $68 \%$ da população entrevistada responderam ser de cor branca, $20 \%$ parda, $7 \%$ indígena, $4 \%$ negra e $1 \%$ amarela. Esse resultado pode ser comparado com dados mencionados por Silva et al. (2011), os quais mostram que 16\% da população que buscou atendimento de saúde em 2003 e 2008, segundo dados da Pesquisa Nacional por Amostras de Domicílios (PNAD), eram brancos. Ou seja, da população que buscou os atendimentos de saúde nesses anos mencionados, os brancos também representaram a maioria, seguido de $14 \%$ de negros e pardos.

Percebeu-se uma maior concentração (67\%) da utilização do serviço de saúde em duas cidades e de 33\% em outras 12 cidades de Santa Catarina. Apenas 5\% reside no centro de suas cidades e o restante ( $85 \%$ ) reside em outros bairros. Quando perguntado há quanto tempo moravam em sua cidade atual, obteve-se uma média de 17 anos, com desvio padrão de 4 anos, sendo que o mínimo de tempo residindo na cidade foi de 1 ano e o máximo de 63 anos.

Segundo Lima et al. (2007), os fatores tempo, distância do percurso e forma de deslocamento podem facilitar ou dificultar o acesso aos serviços de saúde. Para esses autores, quando há a necessidade de deslocamento maior para procurar os serviços em outra cidade, o motivo principal é que a população deseja ter um acesso facilitado e melhor atendimento. As próprias experiências negativas, vivenciadas em suas cidades, motivam as pessoas a procurarem outros lugares que atendam às suas expectativas. Por essa razão, percebe-se também um número significativo de pessoas que procuram os serviços de saúde onde foi realizada a pesquisa, mesmo não residindo tão próximo ao local.

Quanto ao estado civil, $47 \%$ dos entrevistados eram casados; $22 \%$, solteiros; $16 \%$ declararam-se estar em união estável; $8 \%$ eram viúvos e 7\%, divorciados. Este número significativo de pessoas casadas se relaciona com o fato que a relação conjugal dos país é importante para o estado emocional dos filhos, como explica Feres-Carneiro (2008), afirmando que é importante 
a boa relação entre os pais para um melhor apoio aos filhos, principalmente se estes estiverem doentes. Portanto, o casamento dos pais pode influenciar positiva ou negativamente a saúde da criança internada.

Sobre a renda familiar, $51 \%$ respondeu ter renda familiar entre dois e três salários mínimos; $19 \%$, entre três e quatro salários mínimos; $16 \%$, apenas um salário mínimo e 13\% tem renda familiar de mais de quatro salários mínimos. A média do número de pessoas na família, ou seja, que residem na mesma casa que os entrevistados, é de 4 pessoas, com desvio padrão de 1,5, o mínimo de pessoas na família é de 2 pessoas e o número máximo encontrado foi de 13 pessoas.

Para Feliciano, Moraes e Freitas (2004), as pessoas de baixa renda têm maior risco de ficarem doentes, como os idosos e as crianças. Porém, ao contrário do que a literatura traz, os achados quanto à renda familiar dos que utilizavam os serviços de saúde não são de pessoas com a menor renda. Isso pode ser explicado pelo aumento do número de mulheres, homens e jovens, que residem na mesma casa, trabalharem para ajudar com as despesas da casa, visto que atualmente apenas uma pessoa trabalhando, dependendo do salário que recebe, pode não conseguir sustentar toda a família.

Percebeu-se que entre os entrevistados, 62\% tinha uma profissão, verificando-se que $26 \%$ respondeu ser do lar; 4\%, aposentados; $4 \%$, estudantes; $4 \%$, autônomos. Um dado bastante significativo foi que $91 \%$ da população entrevistada foi do sexo feminino e que se verifica um aumento das mulheres no mercado de trabalho - a não ser em algumas situações, em que há a necessidade de substituir o emprego formal por outras atividades, como dedicar mais tempo ao lar e ao cuidado das crianças, considerando os casos em que elas têm filhos recém-nascidos ou doentes - em contrapartida, Lindo et al. (2007) expõem que o salário recebido por essas cuidadoras faz com que não seja viável pagar um funcionário para prover os cuidados de seus filhos.

Na questão escolaridade, a maioria da população entrevistada respondeu ter o ensino médio completo, sendo este número correspondente a $33 \%$; seguido de $18 \%$ de pessoas com ensino fundamental incompleto; $17 \%$ com ensino médio incompleto; 1 1\% com ensino superior incompleto; $10 \%$ possuindo ensino fundamental completo; $6 \%$ com ensino superior completo.

Para Araujo et al. (2012), é importante conhecer o nível de escolaridade dos cuidadores, pois estes recebem informações importantes da equipe de saúde quanto aos cuidados que se têm que ter com o internado, sobre a patologia do mesmo, entre outras. Segundo o autor, a pouca escolaridade poderia influenciar na aprendizagem, dificultando a compreensão do que acontece com o paciente.

\section{VÍNCULO COM O HOSPITALIZADO}

A doença e hospitalização de uma criança afetam todos os membros da família e altera toda a dinâmica familiar, pois ao vivenciar a doença do filho, a família ingressa em um mundo novo, o mundo do hospital, cuja organização, dinâmica e lógica são muito diferentes de seu cotidiano, acarretando um elevado nível de estresse e ansiedade, principalmente quando a criança necessita de cuidados intensivos. Assim, a intensidade deste sofrimento pode apresentar variações de acordo com a doença, evolução clínica e prognóstico (DIAS; MOTTA, 2004; RIBEIRO, 2004).

$\mathrm{O}$ adoecimento da criança é algo muito doloroso e angustiante para seus familiares, pois ela desempenha um papel muito importante no mundo da família, já que representa o futuro, a 
concretização dos sonhos e dos anseios dos pais, que passam a ver as possibilidades do filho como as suas próprias expectativas. A doença do filho, então, rompe com todos estes significados e com os projetos de futuro, acarretando um imenso sofrimento e estresse familiar (DIAS; MOTTA, 2004).

Atualmente, reconhece-se a importância da permanência dos pais no hospital e de seu envolvimento no processo saúde-doença-cuidado da criança. Tal consideração fundamenta-se nos trabalhos da psicologia, os quais apontam os problemas relacionados à separação da família no período de hospitalização (COLLET; ROCHA, 2003).

No cotidiano hospitalar, observa-se que, na maioria das vezes, a mãe é quem permanece junto ao filho durante todo o período da internação, ou quem vem visitá-lo com maior frequência. Quando se consegue estreitar o relacionamento com essas mães, nota-se que elas nutrem um intenso desejo de cuidar do filho durante o processo de internação, como uma forma de transmitir todo o seu amor, aumentar o vínculo mãe-filho e minimizar o sentimento de culpa, muitas vezes presente em face do adoecimento da criança (LIMA; ROCHA; SCOCHI, 1999).

Muito se tem falado em estratégias para incentivar a mãe a participar do cuidado ao filho hospitalizado, mas as práticas assistenciais brasileiras, na grande maioria das instituições, não incorporaram sistematicamente a mãe ou o responsável neste cuidado devido ao despreparo da equipe de profissionais, a qual ainda resiste muito a aceitar a presença da família da criança. Quando ocorre a inserção da mãe no cuidado, isto se faz de maneira inadequada, pois os profissionais delegam à mãe, durante a hospitalização da criança, atividades simples ou por vezes complexas, sem orientação e supervisão adequadas, pois o intuito desta ação fica restrito a delegar/repassar tarefas (LIMA; ROCHA; SCOCHI, 1999; COLLET; ROCHA, 2003).

Assim, embora tais cuidados façam parte do cotidiano hospitalar e componham o repertório de habilidades dos profissionais, todo e qualquer tipo de procedimento representa algo muito novo e abstrato para a população leiga, da qual provém grande parte dos usuários do sistema de saúde. Além disto, é preciso considerar que o momento de fragilidade da mãe/cuidadora costuma intensificar as suas dificuldades de compreensão.

\section{DADOS SOBRE A SAÚDE DO CUIDADOR}

Quanto ao histórico de doença familiar, após a Diabetes Mellitus, as doenças mais citadas foram: Doenças Cardiovasculares, com 24\%; em seguida, a Hipertensão, com 21\% e por fim o Câncer, com 19\%.

Estes dados supracitados podem ser relacionados com os obtidos pelo Ministério da Saúde e publicados na cartilha "Plano de Ações Estratégicas para o Enfrentamento das Doenças Crônicas Não Transmissíveis (DCNT) no Brasil 2011-2022” (BRASIL, 201 1b), que apontam que as doenças respiratórias crônicas, hipertensão arterial, câncer, diabetes, infarto e acidente vascular cerebral são as doenças que constituem o problema de saúde de maior magnitude no Brasil e correspondem a cerca de $70 \%$ das causas de morte no país.

Com relação à prática de exercícios físicos, após a caminhada, os exercícios físicos mais praticados foram: andar de bicicleta (21,62\%); jogar futebol (8,10\%); frequentar a academia $(5,40 \%)$; e ginástica laboral, aulas de jump e natação (todos com 2,70\%).

As implicações positivas, tanto na esfera física quanto na esfera psíquica e mental, que a prática de exercício físico pode ter na vida da população já são bastante conhecidas, porém grande 
parte dos cuidadores entrevistados não possui o hábito de exercitar-se. É difícil dizer se este dado está diretamente relacionado ou não com a enfermidade da criança, sendo que existem diversas possíveis causas para falta de prática de exercício físico, porém se sabe que a saúde e os hábitos de saúde físicos e mentais do cuidador são fortemente influenciados pelo comportamento apresentado pela criança e pela demanda de cuidado que ela apresenta (CAMARGOS et al., 2009).

Em relação ao uso de bebida alcoólica, as alternativas que apresentavam mais frequência de uso ("quatro vezes por semana” e "mais de quatro vezes por semana”) não foram escolhidas nenhuma vez. É possível comparar esses dados com a pesquisa de Fernandes e Garcia (2009), na qual o uso da substância alcoólica, assim como o tabagismo, teve pouca significância, sendo que pouco dos cuidadores entrevistados eram tabagistas, ou passaram a ser tabagistas quando assumiram o papel de cuidador, porém esta mesma pesquisa evidenciou que um número significativo de cuidadores utilizava medicamentos para dormir ou calmantes.

Como relação ao uso do tabaco, o resultado da presente pesquisa ( $14 \%$ dos entrevistados são tabagistas) reflete os dados divulgados pelo Ministério da Saúde, Instituto Nacional de Câncer e Organização Pan-Americana da Saúde (BRASIL, 2011a), os quais afirmam a prevalência de uso do tabaco no Brasil é de 17,5\% da população.

Dos cuidadores que afirmaram utilizar medicamentos de uso contínuo, $41 \%$ relatou fazer uso de anti-hipertensivos. Acredita-se que estes dados estejam relacionados ao fato de os antihipertensivos serem utilizados para tratamento da hipertensão arterial sistêmica, e esta constituir a mais frequente doença cardiovascular e o problema mais relevante de saúde pública no Brasil e no mundo (BRASIL, 2010). Em segundo lugar, na presente pesquisa, aparecem os anticoncepcionais (contraceptivos) e os antidepressivos, com 19\%.

\section{CONSIDERAÇÕES FINAIS}

Por meio dos dados coletados e analisados com os 100 cuidadores de crianças e adolescentes internados no hospital infantil, foi possível perceber a atribuição do papel de cuidador à mulher, principalmente às mães, considerando que $91 \%$ dos cuidadores eram do sexo feminino. Muitas dessas cuidadoras não realizam nenhuma atividade profissional (30\%), sendo que outra parcela significativa das entrevistadas (18\%), mesmo sendo consideravelmente jovens, possui baixa escolaridade (ensino fundamental incompleto). Assim, fica explícito que a mulher ainda assume a função social de dona de casa, responsável pelo cuidado dos filhos e da família. Fica visível, também, que apesar da mãe se apresentar como principal cuidadora, o adoecimento da criança é um fator que mobiliza outros membros da família, principalmente os avós, observando-se que o papel de cuidador é desempenhado com menor frequência pelo pai.

Dessa forma, considera-se a importância de estimular o pai a responsabilizar-se pelos cuidados do filho, enfatizando a importância da presença de ambos para o bem-estar e a recuperação da criança durante sua internação, visto que a presença dos pais é de grande importância para a recuperação da criança ou do adolescente hospitalizado. A participação dos pais no processo saúde e doença evita ou diminui sentimentos de insegurança, medo, angústia, estresse e de abandono pela separação temporária da família.

Quanto aos dados relacionados com o estado de saúde desses cuidadores, é possível considerar o fato da maioria de os entrevistados não praticar nenhum tipo de exercício físico (63\%) 
e possuir histórico de doença familiar (61\%), principalmente diabetes (36\%). Por outro lado, como aspectos positivos, a grande maioria afirma não fazer uso de bebidas alcoólicas (80\%) e nem ser tabagista $(86 \%)$, porém uma parcela significativa (28\%) faz uso de medicamentos de uso contínuo, entre os quais os antidepressivos aparecem com maior frequência.

A maior parte dos cuidadores considera sua saúde como "boa”, ainda assim fica evidente a necessidade de traçar estratégias e desenvolver projetos de atenção e promoção à saúde desses cuidadores, principalmente no que diz respeito à promoção de hábitos de vida adequados e saudáveis, sendo que essas estratégias devem ser de acesso gratuito, estando inseridas no sistema público de saúde, considerando que a grande maioria dos entrevistados afirmou ter renda familiar entre dois e três salários mínimos, outros 16\% alegam renda de até um salário mínimo em contrapartida com $32 \%$ que recebem mais do que três salários mínimos mensais.

Conclui-se também a importância da realização de mais pesquisas na área, para implementação de estratégias de intervenção adequadas a essa população, visto que se faz importante a criação de programas de atenção à saúde dos cuidadores.

REFERÊNCIAS

ARAUJO, J. S.; SILVA, S. E. D. da; SANTANA, M. E. de; CONCEIÇÃO, V. M. da; VASCONCELOS, E. V. O perfil representacional dos cuidadores de pacientes acometidos por acidente vascular cerebral. Revista Eletrônica Gestão \& Saúde, Brasília, v. 3, n. 3, p. 852-864, 2012.

BECK, A. R. M.; LOPES, M. H. B. de M. Tensão devido ao papel de cuidador entre cuidadores de crianças com câncer. Revista Brasileira de Enfermagem, Brasília, v. 60, n. 5, p. 670-675, nov./dez. 2007.

BRASIL, Ministério da Saúde. Formulário terapêutico nacional 2010: Rename 2010. 2. ed. Brasília: Ministério da Saúde, 2010. Disponível em: <http://portal.saude.gov.br/portal/arquivos/pdf/FTN_2010. pdf> Acesso em: 17 mar. 2013.

BRASIL, Ministério da Saúde. Pesquisa especial de tabagismo - PETab: relatório Brasil. Rio de Janeiro: INCA, 2011a.

BRASIL, Ministério da Saúde. Plano de ações estratégicas para o enfrentamento das doenças crônicas não transmissíveis (DCNT) no Brasil 2011-2022. Brasília: Ministério da Saúde, 2011 b.

BRASIL, Ministério da Educação. Programa de Extensão Universitária- PROEXT. Brasília, 2003.

CAMARGos, A. C. R.; LACERDA, T. T. B. de; VIANA, S. O.; PINTO, L. R. A.; FOnSECA, M. L. S. Avaliação da sobrecarga do cuidador de crianças com paralisia cerebral através da escala Burder Interview. Revista Brasileira de Saúde Materno Infantil, Recife, v. 9, n. 1, p. 31-37, jan./mar. 2009.

CAPRARA, A.; FRANCO, L. S. A. A relação paciente-médico. Para uma humanização da prática médica. Cadernos de Saúde Pública, Rio de Janeiro, v. 15, n. 3, p. 647-654, 1999.

CÔA, T. F.; PETTENGILL, M. A. M. A experiência de vulnerabilidade da família da criança hospitalizada em Unidade de Cuidados Intensivos Pediátricos. Revista da Escola de Enfermagem, São Paulo, v. 45, n. 4, p. 825-832, 2011.

COLlET, N.; ROCHA, S. M. M. Participação e autonomia da mãe no cuidado ao filho hospitalizado. Revista Brasileira de Enfermagem, Brasília, v. 56, n. 3, p. 260-264, maio/jun. 2003.

DIAS, S. M. Z.; MOTTA, M. da G. C. Prática e saberes do cuidado de enfermagem à criança hospitalizada. 
Ciência, Cuidado e Saúde, Maringá, v. 3, n. 1, p. 41-54, jan./abr. 2004.

FELICIANO, A. B.; MORAES, S. A. de; FREITAS, I. C. M. de. O perfil do idoso de baixa renda no Município de São Carlos, São Paulo, Brasil: um estudo epidemiológico. Cadernos de Saúde Pública, Rio de Janeiro, v. 20, n. 6, p. 1575-1585, nov./dez., 2004.

FERES-CARneiro, T. Pesquisa e Prática Clínica: construindo articulações teóricas. Psicologia: Reflexão e Crítica, Rio de Janeiro, v. 21, n. 3, p. 349-355, 2008.

FERNANDES, M. das G. M.; GARCIA, T. R. Atributos da tensão do cuidador familiar de idosos dependentes. Revista da Escola de Enfermagem, São Paulo, v. 43, n. 4, p.818-824, 2009.

FLECK, M. P. de A.; LEAL, O. F.; LOUZADA, S.; XAVIER, M.; CHACHAMOVICH, E.; VIEIRA, G.; SANTOS, L. dos; PINZON, V. Desenvolvimento da versão em português do instrumento de avaliação de qualidade de vida da OMS (WHOQOL-100). Revista Brasileira de Psiquiatria, São Paulo, v. 21, n. 1, p. 19-28, jan./mar., 1999.

HAINES, C.; CHILDS, H. Parental satisfaction with paediatric intensive care. Paediatric Nursing, v. 17 , n. 7, p. 37-41, set., 2005.

LIMA, R. A. G.; ROCHA, S. M. M.; SCOCHI, C. G. S. Assistência à criança hospitalizada: reflexões acerca da participação dos pais. Revista Latino-Americana de Enfermagem, Ribeirão Preto, v. 7, n. 2, p. 33-39, abr. 1999.

LIMA, M. A. D. da S.; RAMOS, D. D.; ROSA, R. B.; NAUDERER, T. M.; DAVIS, R. Acesso e acolhimento em unidades de saúde na visão dos usuários. Acta Paulista de Enfermagem, São Paulo, v. 20, n. 1, p. 12-17, jan./mar., 2007.

LINDO, M. R.; CARDOSO, P. M.; RODRIGUES, M. E.; WETZEL, U. Vida Pessoal e Vida Profissional: os Desafios de Equilíbrio para Mulheres Empreendedoras do Rio de Janeiro. RAC-Eletrônica, Rio de Janeiro, v. 1, n. 1, p. 1-15, jan./abr., 2007.

MACHADO, H. B.; SOUZA, M. G. de; MACHADO, C.; REBELLO, B. Percepção de familiares de crianças internadas sobre o papel dos cuidadores. Família, Saúde e Desenvolvimento, Curitiba, v.8, n.1, p.49-55, jan./abr., 2006.

MOREIRA, P. L.; ANGELO, M. Tornar-se mãe de criança com câncer: construindo a parentalidade. Revista Latino-Americana de Enfermagem, Ribeirão Preto, v. 16, n. 3, p. 355-361, maio/jun., 2008.

NETO, A. C. As fases turbulentas da hospitalização. Revista Pediatria Moderna, v. 4, n. 36, p. 245-247, 2000.

ORTIZ, M. C. A.; LIMA, R. A. G. de. Experiences of families with children and adolescents after completing a cancer treatment: support for the nursing care. Revista Latino-Americana de Enfermagem, Ribeirão Preto, v. 15, n. 3, p. 411-417, maio/jun., 2007.

RIBEIRO, N. R. R. A família enfrentando a doença grave da criança. In: ELSEN, I.; MARCON, S. S.; SILVA, M. R. S. da. (Org.) O viver em família e sua interface com a saúde e a doença. 2. ed. Maringá: EDUEM, 2004. p. 183-197.

SILVA, Z. P. da; RIBEIRO, M. C. S. de A.; BARATA, R. B.; ALMEIDA, M. F. de. Perfil sociodemográfico e padrão de utilização dos serviços de saúde do Sistema Único de Saúde (SUS), 2003- 2008. Ciência \& Saúde Coletiva, Rio de Janeiro, v. 16, n. 9, p. 3807-3816, set., 2011.

VASCONCELOS, E. M. Redefinindo as práticas de saúde a partir da educação popular nos serviços de saúde. In: VASCONCELOS, E. M. (Org.) A saúde nas palavras e nos gestos: reflexões da rede de educação popular e saúde. São Paulo: HUCITEC, 2001. p.11-9. 